\title{
Evolutionary Spatial Games Under Stress
}

\author{
J. Alonso ${ }^{1}$, A. Fernández ${ }^{1}$, and H. Fort ${ }^{2}$ \\ ${ }^{1}$ Universidad de la República, Institute of Physics, Facultad de Ingeniería, Julio \\ Herrera y Reissig 565, 11300 Montevideo, Uruguay \\ ${ }^{2}$ Universidad de la República, Institute of Physics, Facultad de Ciencias, Iguá 4225, \\ 11400 Montevideo, Uruguay
}

\begin{abstract}
We analyse different evolutionary spatial games, in which the pressure of the environment is taken into account, using binary cellular automata. The agents are unconditional players: at each time step a given cell cooperates (play C) or defects (play D) against all its neighbours. The pressure of the environment is implemented by requiring a minimum score $U_{\text {min }}$, representing indispensable resources (nutrients, energy, revenues, etc.) for an individual to prosper. Therefore a cell, instead of evolving just by adopting the state of its most successful neighbour, also takes into account if the "winner" gets a score above or below $U_{\min }$. In the latter case it has a probability of adopting the opposite state. Besides the paradigmatic and widely used Prisoner's Dilemma (PD), two other games are analysed: the Hawk-Dove (H-D), popular in biology, and the Stag Hunt (SH) that recently came into favour in social sciences. The effect of the environmental stress is particularly dramatic in the case of the PD: it allows the evolution of cooperation for payoff matrices where defection was the rule for simple unconditional strategy players. Finally, we discuss a more sophisticated model version in which the ordinary evolutionary recipe of copying the most successful neighbour is supplemented with a "win-stay, lose-shift" criterion. This model variant, for a restricted region of the parameter space, produces critical scaling laws.
\end{abstract}

\section{Introduction}

Cooperation and competition in nature represent two faces of a same coin. Progress seems to need both: competition promotes efficiency at the individual level but cooperation is indispensable to achieve collective goals.

Game theory is a branch of mathematics concerned with the analysis of conflict situations. Social dilemma games demonstrated to be a suitable approach to describe this tension between individual and global interests in social sciences [1] as well in biology [2].

In social dilemma games each individual ("player") receives a higher payoff for a socially defecting choice than for a socially cooperative choice no matter what the other individuals in society do. But, at the same time, all individuals would be better off if all cooperate than if all defect. The most popular of such games is the Prisoner's Dilemma (PD) [3]. Imagine two players, each confronting two choices: cooperate (C) or defect (D), and each makes his choice without 
knowing what the opponent will do. The possible outcomes are: 1) they can both cooperate: $(\mathrm{C}, \mathrm{C})$ and get the "reward" for mutual cooperation $R, 2)$ they can both defect: (D,D) and get the "punishment" $P$ for mutual defection or 3) one of them cooperates and the other defects: $(\mathrm{C}, \mathrm{D})$; in that case the one who played C gets the "sucker's payoff" $S$ while his opponent gets the "temptation to defect" $T$. Hence we have a $2 \times 2$ payoff matrix with the payoffs obeying the chain of inequalities $T>R>P>S$. So independently of what the other player does, defection $\mathrm{D}$ yields a higher payoff than cooperation $\mathrm{C}$ : if your opponent defects, and you cooperate you will end up with the worst payoff. On the other hand, even if your opponent cooperates, you should defect because in that case your payoff is $T$ which is higher than $R$. Nevertheless, playing both $\mathrm{D}$ they get $P$ which is worst than $R$.

A more interesting situation arises when the game is played repeatedly. In this iterated Prisoner's Dilemma (IPD), if players have memory (at least from the immediately previous contest), there are several strategies that outperform the dominant one-shot strategy $[\mathrm{D}, \mathrm{D}]$ and lead to some non-null degree of cooperation. For instance, in the strategic tournaments organised by Axelrod [1, [4] in the 80s, the winner strategy was 'TIT FOR TAT' (TFT), which plays C on the first move, and on all subsequent moves copy the choice of its opponent on the previous move. Afterwards a different computer tournament was carried out by Nowak and Sigmund [6. In that case the winner strategy was the one previously named simpleton by Rapoport and Chammah [7 or Pavlov by D. and V. Kraines [8, because if its action result in a high payoff $(T$ or $R$ ) it keeps it, but otherwise changes it.

On the other hand, territoriality by itself provides an alternative way to escape from iterated evolutionary game dilemmas without resorting to memory of past encounters conditioning the behaviour, which are conditional strategies (like TFT, Pavlov, etc.). This was shown by Nowak and May [9] who proposed a cellular automaton in which agents are binary unconditional strategists, that is: at each time step a given cell play versus all of their neighbours either C or D. In the next generation, a given cell adopts the state of the most successful cell of the neighbourhood (the one that collected the highest score among the cell itself and its neighbours). The game they considered is a simplified version of the PD in which the punishment $P$ and the sucker's payoff $S$ are equal 1 , implying then a "weak dilemma" (maximum punishment for mutual defection). Specifically they fixed $R=1$ and $P=S=0$ and found that the fraction of cooperators $c$ varies from 1 to 0 as the temptation increases from $T=1$ to $T=2$. However, a problem with this simple model is that $c$ drops to zero for ordinary non weak dilemmas in which all the individuals end playing $\mathrm{D}$.

In the present work we propose a simple extension of the unconditional binary C.A. that allows the evolution of cooperation not just for weak dilemmas but for ordinary ones, where $P$ and $S$ can be quite different. The basic idea is that individuals need to collect, when playing with their $z$ neighbours, a score

\footnotetext{
${ }^{1}$ Indeed this game is the frontier between the PD and the game known as chicken (see below).
} 
above certain threshold $U_{\min }$ in order to prosper. In an ecosystem $U_{\min }$ represents the minimal resources (nutrients, energy, etc.) for surviving, alternatively, in markets it may represent the minimum revenues that make investments profitable. Provided the difference between $P$ and $S$ is sufficiently large, defecting individuals are on average the most successful in the first rounds. Nevertheless, if $U_{\min }>z P$, they perform very poorly when surrounded by an entire neighbourhood of D's (something that in the real world this may be equivalent to death). Therefore we include a probability $p$ of adopting the state opposite to the one of the most successful neighbour when its score is below $U_{\min }$.

In order to minimise the number of model parameters we use a two-parameter payoff matrix and we fix the worst payoff $X^{(4)}$ to 0 and the second best $X^{(2)}$ to 1 (for example $S=0$ and $R=1$ for the PD). We explore a subspace of the space of parameters $\left\{T, P, U_{m i n}, p\right\}$ characterising the $\mathrm{PD}$ and measure the asymptotic (after a transient) fraction of cooperators. Furthermore, changing the rank order of the 4 payoffs we explore two other dilemma games. First, when the damage from mutual defection in the PD is increased so that it finally exceeds the damage suffered by being exploited, i.e. $T>R>S>P$, the new game is called the chicken or Hawk-Dove (H-D) game [2]. The 'Hawk' and 'Dove' allude to the two alternative behaviours displayed by animals in confrontation: hawks are expected to fight for a resource and will injure or kill their opponents (i.e. play D), doves, on the other hand, only bluff and do not engage in fights to the death (i.e. play C). This game applies thus to situations such that mutual defection (Hawk vs. Hawk) is the worst possible outcome for both as it happens in most of animal contests. Second, a nowadays popular game in social sciences is the Stag Hunt [10], corresponding to the payoffs rank order $R>T>P>S$ i.e. when the reward for mutual cooperation surpasses the temptation to defect. The name of the game derives from a metaphor invented by the French philosopher Jean Jacques Rousseau: Two hunters can either jointly hunt a stag or individually hunt a rabbit. Hunting stags is quite challenging and requires mutual cooperation. If either of them hunts a stag alone, the chance of success is minimal. Hunting stags is most beneficial for society but requires trust among its members.

\section{The Model}

The individuals (people, firms, bacteria, etc.) are represented by cells of a twodimensional binary cellular automaton (CA). That is, each cell can take two possible values 0 or 1 corresponding to unconditional strategies for playing versus their neighbours: cooperate $(\mathrm{C})$ or defect $(\mathrm{D})$.

In this work we restrict ourselves to a) the von Neumann neighbourhood $(z=4$ neighbouring cells: the cell above and below, right and left from a given cell) and b) the Moore neighbourhood ( $z=8$ neighbouring cells surrounding a given cell). Typical grid sizes range from $50 \times 50$ to $500 \times 500$. Periodic boundary conditions are used. The score of a given player is the sum of all the payoffs he gets against each neighbour. The dynamic is synchronous: all the agents update their states simultaneously at the end of each CA-step. 
In the CA of Ref. 9] natural selection is implemented very simply: each player adopts the strategy of the most successful neighbour (who got $U^{m s n}$ ). Here, we consider the following modified evolutionary rule: If $U^{m s n}>U_{m i n}$, then the player adopts the strategy of the most successful neighbour in the next generation. Otherwise, the player has a small probability $p$ of adopting the opposite strategy. The rationale for this is that copying the most successful neighbour, when its payoff doesn't reach a critical threshold, may not be the most efficient strategy from an evolutionary point of view.

\section{Results}

After a transient the system reaches a steady state with a definite value $c$ for the fraction of agents playing $\mathrm{C}$. The duration of the transient depends on the lattice size and the neighbourhood. For instance for a $50 \times 50$ lattice and $z=8$ it last typically between 100 and 200 rounds.

To avoid dependence on the initial conditions, the measures correspond to averages over an ensemble of 100 systems 2 with arbitrary conditions.

Here, we present results for a subspace of the parameter space: the third best payoff is fixed to $X^{(3)}=0.5$. This value is chosen such that in the case of the PD, a punishment of $P=0.5$ implies, without any doubt, a non weak dilemma 3 . We studied several values of the probability parameter and the ones reported here are always for $p=0.1$. The best payoff $X^{(1)}$ is varied between 1 and 2. Finally, since $U_{\min }<z X^{(3)}$ has no effects and $U_{\min }>z X^{(1)}$ doesn't make sense (since no one can reach this threshold), the parameter space reduces to the square plane $X^{(1)}-U_{\min }$ delimited by $1 \leq X^{(1)} \leq 2$ and $z X^{(3)} \leq U_{\min } \leq z X^{(1)}$.

\subsection{Prisoner's Dilemma: $S=0, P=0.5, R=1$ and $1<\mathrm{T} \leq 2$}

Figs. 1, a and 1] b show $c$ as a function of $T$ and $U_{\min }$. Basically three regions can be distinguished in the plots: i) A stepladder region emerges from the right border $U_{\min }=z P$. ii) For not too large values of $T$ and $U_{\min }$ there is a high peak of cooperation, delimited at the left by $U_{\min }=z R=z$ (when all the cells play C). iii) Finally, beyond $U_{\min }=z R=z c$ reaches a plateau delimited by the straight line $U_{\min }(T)=z T$.

To understand the 3 different regions it is convenient to consider a small deviation from the minimum $T: T=1+\epsilon$. Therefore, for $z=8$ and $P=0.5$ Table 1 summarises the different payoffs for a player depending on the number of C's and D's in its neighbourhood.

Let's start with the peak. For $U_{\min }$ greater than 6 , only D's surrounded by at least $4 \mathrm{C}$ 's can achieve the minimum $U_{m i n}$, so cooperation grows dramatically. This corresponds to $\epsilon \lesssim 0.16$, (i.e. $T \lesssim 1.16$ ). If $U_{\min }=8$ then $c$ drops abruptly since even $\mathrm{C}$ agents surrounded entirely by other C's cannot survive anymore.

$\overline{2}$ The relative error in these averages is about 7 percent.

${ }^{3}$ Both for $z=4$ or $z=8, P=0.5$ leads to $c=0$ when simulating the model of Ref. 9 . 
Table 1. Score of a player depending on its state $\mathrm{C}$ or $\mathrm{D}$ and the number of $\mathrm{C}$ and $\mathrm{D}$ agents in its $z=8$ neighbourhood for $T=1+\epsilon$ and $P=0.5$

\begin{tabular}{|c|c|c|c|c|c|c|c|c|c|}
\hline & $8 C, 0 D$ & $7 C, 1 D$ & $6 C, 2 D$ & $5 C, 3 D$ & $4 C, 4 D$ & $3 C, 5 D$ & $2 C, 6 D$ & $1 C, 7 D$ & $0 C, 8 D$ \\
\hline$C$ & 8 & 7 & 6 & 5 & 4 & 3 & 2 & 1 & 0 \\
\hline$D$ & $8.0+8 \epsilon$ & $7.5+7 \epsilon$ & $7.0+6 \epsilon$ & $6.5+5 \epsilon$ & $6.0+4 \epsilon$ & $5.5+3 \epsilon$ & $5+2 \epsilon$ & $4.5+\epsilon$ & 4 \\
\hline
\end{tabular}

(a)

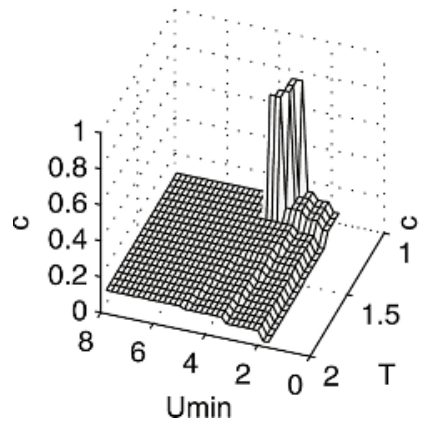

(b)

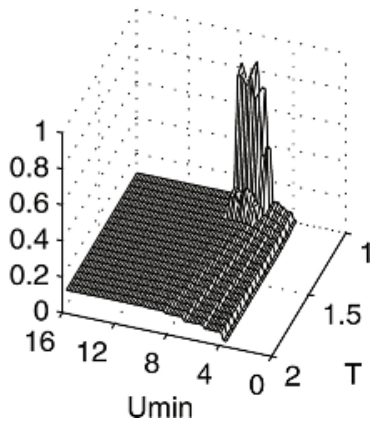

Fig. 1. Fraction of cooperators for $p=0.1$ : (a) $z=4$ neighbours and (b) $z=8$ neighbours

The stepladder structure can be easily explained considering the payoff values for $\mathrm{D}$ agents. As long as $U_{\text {min }}$ increases each $\mathrm{D}$ agent needs more $\mathrm{C}$ agents in its surroundings in order to achieve this threshold. So cooperation grows with $U_{\text {min }}$ by steps at the values: $U_{\min }=T+7 P=4.5+\epsilon, U_{\min }=2 T+6 P=5+2 \epsilon$ and so on, which correspond to straight lines with different slopes in the $\left(T, U_{\min }\right)$ plane .

Finally, when $U_{\min }>8 T$ the minimum required is above any agent's possible score, then the fraction of agents $\mathrm{C}$ one time step further will be given by

$$
c(t+1)=p f_{D}+(1-p) f_{C},
$$

where $f_{D}\left(f_{C}\right)$ stands for the fraction of agents whose most successful neighbour is a D (C). As none of the agents achieves the threshold, everyone with probability $p$ adopts a state opposite to the one adopted by the most successful neighbour. For relatively small values of $p, f_{C} \approx 0$ i.e. $f_{D} \approx 1$ and thus from (1) $c \approx p$. This explains why the height of the plateau coincides with $p$.

\subsection{Hawk-Dove: $P=0, S=0.5, R=1,1<T \leq 2$ and Stag Hunt: $S=0$, $P=0.5, T=1,1<R \leq 2$}

Let's briefly review what happens for these other two games. In Fig. 2] a we observe a qualitatively similar $3 \mathrm{~d}$ plot for $c$ in the case of the H-D. There is a peak in the same place although thicker. On the other hand the SH (see Fig. 2. b) is a much more cooperative than the PD and the threshold has no drastic effects except when $R$ is close to 1 . In that case, once again, there are some intermediate values of $U_{\min }$ that optimise $c$. 

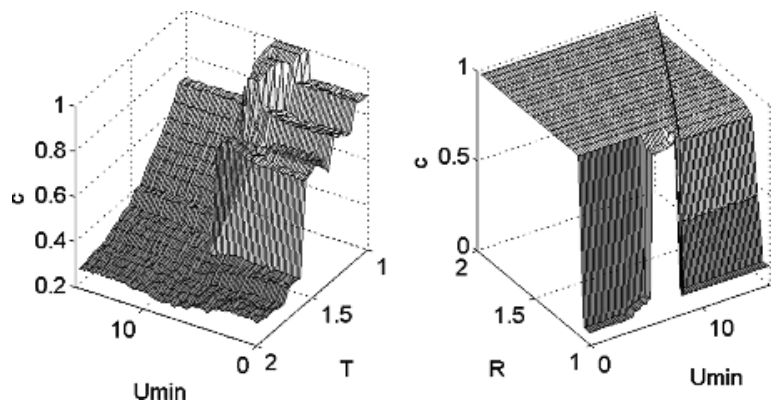

Fig. 2. Fraction of cooperators $c$ for $z=8 \& p=0.1$.(a) H-D (b) SH.

(a)

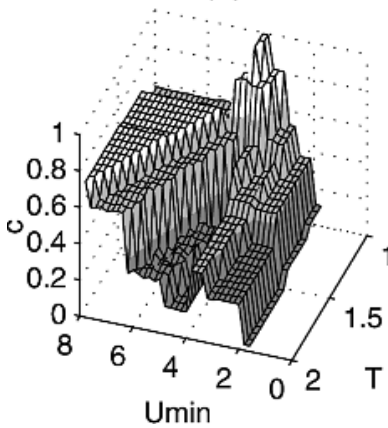

(b)

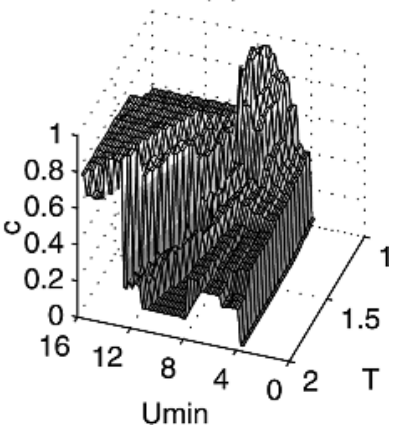

Fig. 3. $c$ for the PD hybrid variant $(p=0.1)$. (a) $z=4$ neighbours and (b) $z=8$ neighbours.

\subsection{Hybrid Version: Natural Selection Complemented with a Pavlovian Criterion}

A relevant input for the behaviour update rule is the comparison of the individual's score with $U_{\min }$. If it is above $U_{\min }$ then the agent's behaviour may be worth keeping even if it is not the most successful neighbour $m s n$. Then we implement this variant as follows: If the cells's score is above $U_{\min }$ it copies the state of the $m s n$ with probability $p$ (or equivalently remains in its state with probability $1-p$ ). If the cells's score is below $U_{\min }$ there are two alternatives: If $U^{m s n}>U_{\min }\left(U^{m s n}<U_{\min }\right)$ the cell copies the state of the $m s n$ (opposite of the $m s n$ ) with probability $1-p$ or remains in its state with probability $p$.

Therefore, this variant interpolates between the ordinary evolutionary recipe of copying the msn and the "win-stay, lose-shift" criterion analysed in detail by Herz [11].

The main modifications in the "landscape" are shown in Fig. 3. Firstly, a strong increase in $c$, for all the parameter space surrounding the peak zone, is observed. Secondly, most part of the plateau is replaced by steep "cliffs". 


\subsection{Cluster Structure}

Spatial patterns were studied for the three games. The most important result deals with the cluster structure of $\mathrm{C}$ agents. It turns out that the PD hybrid model variant exhibits power-law scaling for a very reduced region in the plane $T-U_{\min }$ in the vicinity of the point $\left[T=1.6, U_{\min }=7.5\right]^{4}$. The corresponding histogram of size distribution of clusters is shown in Fig. 4 and fits well with a power law with exponent $-1.6357 \pm 0.0001$. Power laws are the signature of spatial organization of processes into a critical state without any dominating scale.

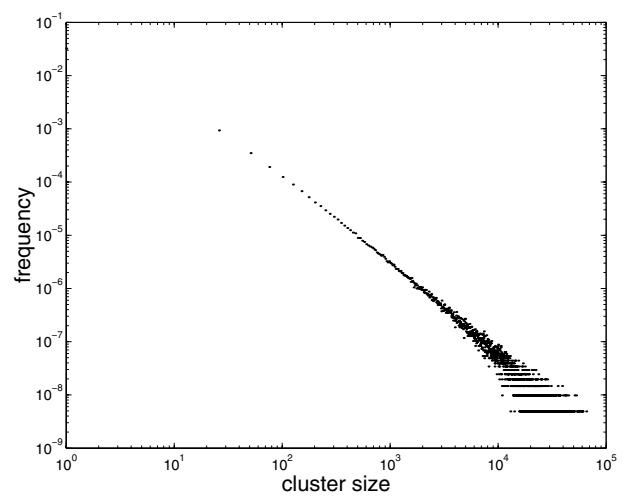

Fig. 4. Dependence of the number of C clusters with size (in logarithmic scale) for the $\mathrm{PD}$ hybrid variant for $T=1.6, U_{\min }=7.5(p=0.1) .(500 \times 500$ lattice $)$

\section{Discussion}

We extended a simple CA model to include, besides space and time (repetition), the influence of the environment exerting pressure on individuals to cooperate. This is done by requiring a minimum score $U_{m i n}$ necessary for agents to carry on vital functions.

This recipe gives rise to cooperation among self-interested individuals from evolution in different dilemma games, involving quite arbitrary payoff matrices. Since we resort to the simplest possible agents - unconditional strategists without long term memory - this allows a potential applicability of the model to a wide variety of contexts from virus (recent experiments indicate that RNA virus may engage in simple two-player games [12]) to social sciences problems.

It is remarkable that, for moderate values of the best payoff, there is an intermediate range of values of $U_{\min }$ that produces a jump in the cooperation for all the three games analysed.

Finally, we introduced and studied a more complex model in which the ordinary evolutionary recipe, of copying the most successful neighbour, is supplemented with a Pavlovian "win-stay, lose-shift" criterion. Besides leading to global

\footnotetext{
${ }^{4}$ The H-D and SH or the basic PD variant don't give rise to power-laws.
} 
optimisation (a much higher cooperation level), this gives rise to power-laws in the size distribution of C-agent clusters. This critical scaling was already found in a different study of CA playing the PD game with Pavlovian strategies [13].

\section{References}

1. Axelrod, R. in: The Evolution of Cooperation, (Basic Books, New York, 1984);

2. Maynard-Smith, J.: Evolution and the Theory of Games, (Cambridge Univ. Press 1982).

3. Flood, M. : Some Experimental Games, Research Memorandum (RAND), RM-789 (1952).

4. Axelrod, R. and Hamilton, W. D. : The evolution of cooperation. Science 211 (1981) 1390-1396

5. Axelrod, R. : J. of Conflict Resolution 24 (1980) 379.

6. Nowak, M.A. and Sigmund, K. : A strategy of win-stay, lose-shift that outperforms tit for tat in Prisoner's Dilemma, Nature 364 (1993) 56-59.

7. Rapoport, A. and Chammah, A.M. :, Prisoner's Dilemma (The University of Michigan Press 1965) pp. 73-74.

8. Kraines, D. and Kraines, V. : Pavlov and the Prisoner's Dilemma, Theory Decision 26 (1988) 47-79.

9. Nowak, M.A. and May, R. : Evolutionary Games and Spatial Chaos, Nature 359 (1992) 826-28.

10. Skyrms, B. :The Stag Hunt and the Evolution of Social Structure, Cambridge University Press 2004.

11. Herz, A.V.M. : Collective Phenomena in Spatially Extended Evolutionary Games, J. Theor. Biol. 169 (1994) 65-87.

12. Turner, P.E. and Chao, L. : Prisoners Dilemma in an RNA Virus, Nature 398 (1999) 441-443.

13. Fort, H. and Viola, S. : Spatial patterns and scale freedom in Prisoner's Dilemma cellular automata with Pavlovian strategies, J. Stat. Mech. (2005) P01010. 